\title{
The feasibility, acceptability and outcomes of exergaming among individuals with cancer: a systematic review
}

\author{
Daniel Tough ${ }^{1 *}$ D, Jonathan Robinson ${ }^{1}$, Steven Gowling ${ }^{2}$, Peter Raby ${ }^{1}$, John Dixon ${ }^{1}$ and Samantha L. Harrison ${ }^{1}$
}

\begin{abstract}
Background: Individuals with cancer have reduced quality of life, functionality, range of motion, strength, and an increase in pain and fatigue. Exergaming appears to be an effective rehabilitation tool for Parkinson's disease, multiple sclerosis and post-stroke patients to improve functionality, balance and quality of life; however, the usefulness of exergaming in individuals with cancer is unknown. The aim of this systematic review is to describe exergaming interventions delivered to adults with a current or previous cancer diagnosis and to report the feasibility, acceptability and outcomes of such interventions.

Methods: Studies reporting on exergaming interventions delivered to individuals with a current or previous cancer diagnosis were included. 12 electronic databases were searched. Eight articles (seven interventions) were identified. Data were extracted and assessed for quality by two reviewers.

Results: Three interventions were delivered at hospital, two at home, one at a clinical laboratory, and one did not report. Two interventions were delivered by a physiotherapist, two by an occupational therapist, and one by a nurse, research staff and an exercise physiologist. The Nintendo Wii was used in four of seven studies, whilst the remaining three used the IREX system, BrightArm Duo Rehabilitation System or a custom made exergame. Studies showed that most participants enjoyed the exergaming intervention, and would recommend their use, with some preferring exergaming over standard care interventions. Adherence rates and enjoyment appear greater during exergaming than standard care. Exergaming interventions appear to support improvements balance, function, physical activity levels, strength, fatigue, emotions, cognition and pain.

Conclusion: Exergaming interventions delivered to individuals with cancer show great heterogeneity; differing in duration, frequency and gaming platform. The disease stage and severity of those included, and the outcome measures assessed also vary widely making it difficult to conclude its effectiveness at this time. However, adherence rates and enjoyment appear greater during exergaming compared to standard care, supporting the feasibility and acceptability of this type of intervention delivery for adults with cancer.
\end{abstract}

Keywords: Cancer, Exergaming, Active video games, Rehabilitation

\footnotetext{
* Correspondence: d.tough@tees.ac.uk

${ }^{1}$ School of Health and Social Care, Teesside University, Middlesbrough TS1

3BX, UK

Full list of author information is available at the end of the article
}

C The Author(s). 2018 Open Access This article is distributed under the terms of the Creative Commons Attribution 4.0 International License (http://creativecommons.org/licenses/by/4.0/), which permits unrestricted use, distribution, and reproduction in any medium, provided you give appropriate credit to the original author(s) and the source, provide a link to the Creative Commons license, and indicate if changes were made. The Creative Commons Public Domain Dedication waiver (http://creativecommons.org/publicdomain/zero/1.0/) applies to the data made available in this article, unless otherwise stated. 


\section{Background}

Cancer is the second leading cause of deaths worldwide, with 8.8 million cancer-related deaths in 2015 alone [1, 2]. Cancer patients commonly experience symptoms such as cancer-related fatigue (CRF), decreased functionality and range of motion (ROM), decreased strength, pain, insomnia and mood disturbances, leading to a decrease in quality of life (QOL), following surgery or pharmaceutical treatments [3-7]. The American College of Sports Medicine recommend that individuals with cancer should avoid inactivity and return to normal daily activities as soon as possible if they undergo surgery, or continue daily routines and exercise if undergoing non-surgical treatments [8]. The National Comprehensive Cancer Network [9] supports this and recommends starting rehabilitation upon diagnosis, continuing up to, and following, completion of treatment.

A lack of physical activity has been associated with an increase in mortality rates [10-12] and a higher disease recurrence [13]. Previous systematic reviews indicate that exercise interventions, consisting of aerobic and resistance training, delivered to individuals with a diagnosis of cancer significantly alleviates some side-effects of surgery, including CRF, and improves exercise capacity and QOL [14-17]. Adherence to such exercise interventions is poor $(<50 \%$ of prescribed sessions attended on occasions) $[16,18]$, potentially due to exercises being monotonous [19]. Current rehabilitation strategies for those with cancer advocate exercises such as stretching, ROM exercises, fine motor training, yoga, aerobic and resistance training exercises [7, 20]. In one study, supervised exercise sessions had an adherence rate of $73 \%$, compared to only $8.7 \%$ in a home-based program [21]. Adherence to an exergaming intervention has shown to be greater than among those undergoing pulmonary rehabilitation [22] and dementia [23].

Exergaming, defined as the combination of exercise and gaming, is a relatively new intervention idea, whereby the user must use physical movements in order to interact with a game [24]. Such games can be played through hand-held controllers (Nintendo Wii), physical movement captured through video-cameras (Sony EyeToy and Microsoft Xbox Kinect) or weight-sensing platforms (Dance Dance Revolution [DDR] and Nintendo Wii Fit) [25]. Exergaming has been found to be an acceptable method for exercising among older adults $[26,27]$. It has also been found to be safe [28-30], easy to use [26] and enjoyable [26, 27]. Exergaming consoles are relatively inexpensive, with the Nintendo Wii and Microsoft Xbox Kinect currently costing less than $£ 250$ and $£ 300$, respectively. Recent systematic reviews have demonstrated the feasibility, acceptability and effectiveness of exergaming at improving balance, functionality, cognition and QOL in individuals with chronic conditions, including Parkinson's disease (PD) [24], multiple sclerosis (MS) [31], cystic fibrosis [32] and those who have recently suffered a stroke [33]. Exergaming has also been found to be a fun and enjoyable method of physical activity, potentially increasing one's motivation to partake in exercise programs [34]. The benefits of exergaming in other clinical populations are becoming increasingly recognised, however there is limited research assessing the feasibility, acceptability and effectiveness of exergaming interventions amongst patients with a current or previous cancer diagnosis.

To our knowledge, no systematic review has synthesised the evidence of exergaming among adults with a current or previous cancer diagnosis. It is therefore important to review the current literature to examine whether an exergaming intervention can indeed support cancer rehabilitation. It will also help to identify the best mode of intervention delivery for this clinical population, guiding future studies. The aim of this systematic review is three-fold; 1) To describe exergaming interventions applied in individuals with cancer, 2) To assess the feasibility and acceptability of an exergaming intervention delivered to adults (18+ years) with a current or previous cancer diagnosis, and 3) To explore the outcomes of such an intervention in this clinical population. Gathering such evidence in a systematic way may help to inform early exergaming interventions for individuals with cancer, within a hospital or home environment, as an alternative to standard mobilisation and exercise therapy.

\section{Methods}

The systematic review is registered with PROSPERO: CRD42017054615. The reporting of the review is consistent with PRISMA guidelines [35].

\section{Search strategy}

The search strategy was developed by one reviewer (DT) in conjunction with a professional librarian. The titles, keywords and abstracts of each article, where applicable, were searched with set search terms (Appendix 1). $\mathrm{MeSH}$ headings were searched for 'exergaming' and 'cancer', or the nearest available terms, to identify relevant search terms. The references of the included articles were also checked.

The following electronic databases were searched: CINAHL, MEDLINE, AMED, PEDro, Cochrane Library Online, ScienceDirect, SPORTDiscus, EMBASE, ASSIA, Scopus, Nursing and Allied Health Source and PsycINFO. All databases were searched from inception to January 2017.

\section{Screening process}

The main researcher (DT) checked for, and removed, all duplicated publications from the initial search. All titles and abstracts were screened independently by two reviewers (DT and SG/PR). Any publications which the 
reviewers were in disagreement over were resolved through a discussion until a consensus was reached. Upon identifying relevant articles, all reviewers evaluated the full text of these articles against the study's inclusion criteria.

\section{Article selection}

Participants, intervention, comparison, outcomes and study design were used to identify the inclusion and exclusion criteria for the study.

\section{Participants}

Adults ( $\geq 18$ years) with a current or previous diagnosis of cancer at the time of testing, regardless of whether surgery had been performed.

\section{Intervention}

Studies reporting on interventions involving exergames as part of the protocol were included. The exergames must have encouraged exercise and physical activity, in order to interact with the game. Any studies commenting on feasibility and acceptability of such interventions were included.

\section{Comparison}

No comparative groups were required to be included.

\section{Outcomes}

All health outcomes were considered within the review including, but not limited to, CRF, QOL, balance, fitness, strength and ROM. Any feasibility and acceptability findings, including adherence rates, were included.

\section{Study design}

Inclusion was not limited by trial design. Studies using both qualitative and quantitative methods were included within the review. Any reviews of the literature were excluded from the study. All articles were required to be available in English, as well as being published within peer-reviewed journals.

\section{Data extraction}

Data extraction was carried out by one reviewer (DT) before being verified by a second reviewer (SG). Participant characteristics, the disease, equipment used, intervention details such as the frequency, duration and setting, comparison groups, outcome measures and significant findings were all extracted from the chosen articles.

\section{Study quality assessment}

The Cochrane Collaboration's tool was used to assess the quality of any controlled trials [36]. This tool uses seven bias domains to enable a user to judge each aspect as a high, low or unclear risk of bias, allowing readers to critically assess the quality of an intervention [37]. For observational studies, the Quality Checklist for Healthcare Intervention Studies was used [38]. This checklist, consisting of 27 items, has been described as one of the best evaluation checklists available due to its ease of use, high reliability and validity and suitable for systematic reviews [39]. It provides an overall study quality index and includes categories addressing reporting, external quality, internal validity-bias, internal validity-confounding and a power rating [40]. The Critical Appraisal Skills Program (CASP) tool [41] was used for any qualitative studies. The qualitative CASP checklist helps users to review an article based upon 10 questions that rate the credibility, relevance and rigour of the study [42]. It allows for easy identification of what a study is lacking, whilst being able to compare study qualities to one another [43]. Each identified article was assessed for risk of bias with the relevant aforementioned tool by two reviewers (DT and SG) independently, before conferring, with any disagreements resolved through a discussion.

\section{Data analysis}

Upon extracting all appropriate data from relevant journal articles, a descriptive summary was conducted to describe the interventions, assess the feasibility and acceptability of exergaming. The outcomes of the interventions were also described among patients with a current or former diagnosis of cancer.

\section{Results}

A total of 4276 articles were screened (excluding duplicates). 17 full text articles were identified and reviewed. Nine studies were excluded from the review; three didn't involve exergaming, two weren't available in English, in two articles the cancer patients weren't segregated from the other participants, one was a review of the literature which wasn't cancer specific and also didn't involve exergaming and the final article didn't explore the feasibility, acceptability or outcomes of an exergaming intervention (Fig. 1). Of the eight remaining studies, one intervention [44] was a continuation of a previous intervention [45] by the same authors the previous year; therefore, these two were combined so as not to allocate unfair weighting to this study.

\section{Study quality design and assessment}

One randomised controlled trial [46] and one controlled trial were included [20], both of which were assessed for risk of bias using the Cochrane Collaboration's tool. The quality assessment didn't detect a high risk of bias for either study (Table 1).

One study [26] was an exploratory study which was assessed using the CASP tool. Most of the aspects were 


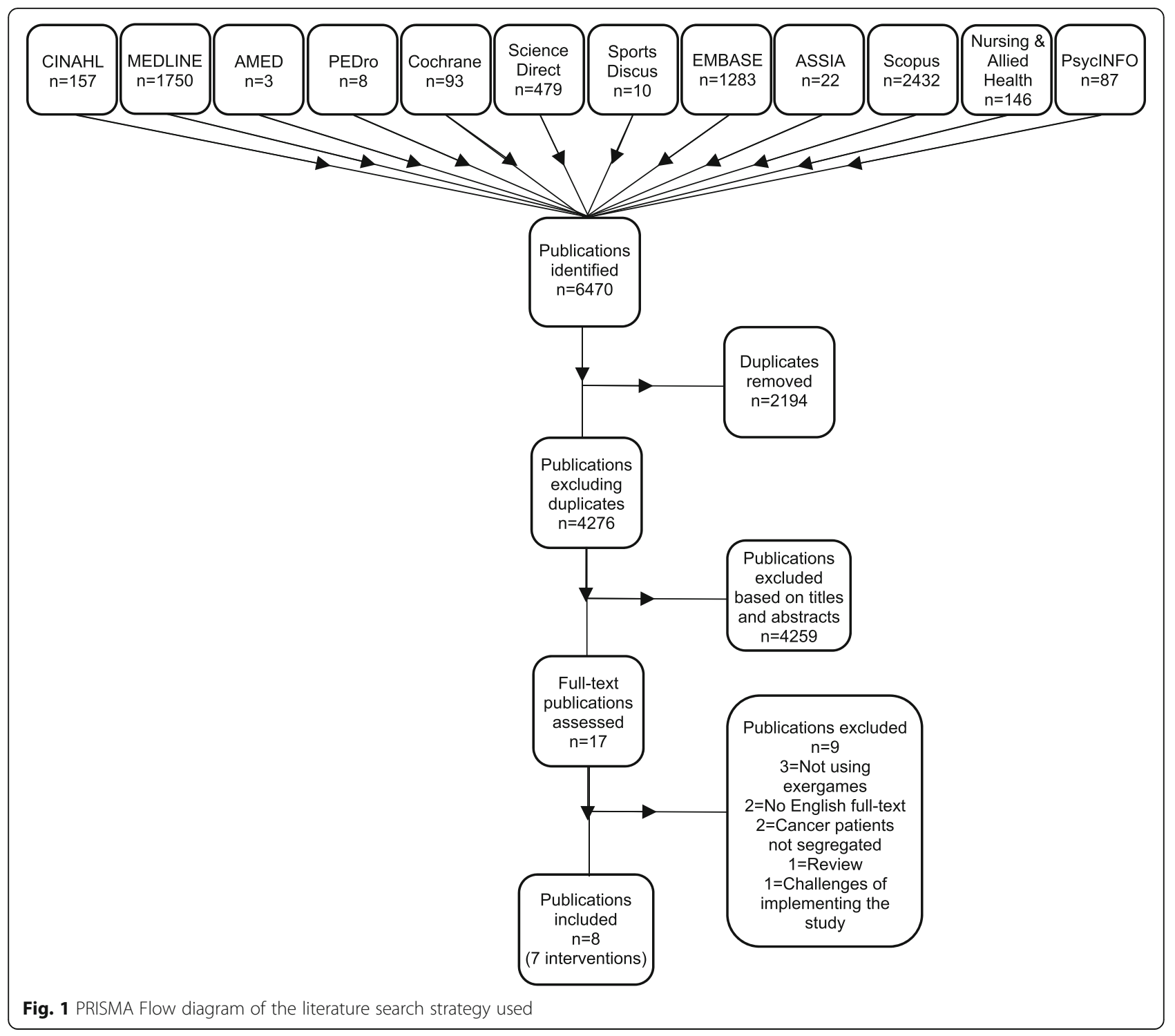

Table 1 Study quality assessment for controlled trials using the Cochrane Collaboration's Tool

\begin{tabular}{|c|c|c|c|c|c|c|c|}
\hline Author (Date) & Selection bias & $\begin{array}{l}\text { Allocation } \\
\text { Concealment }\end{array}$ & Performance bias & Detection bias & Attrition bias & $\begin{array}{l}\text { Reporting } \\
\text { bias }\end{array}$ & Other \\
\hline Sajid, et al. [46] & $\begin{array}{l}\text { Low - Participants } \\
\text { were randomised } \\
\text { into one of three } \\
\text { groups }\end{array}$ & $\begin{array}{l}\text { Unclear - } \\
\text { Randomisation } \\
\text { concealment not } \\
\text { specified }\end{array}$ & $\begin{array}{l}\text { Low - Blinding } \\
\text { of participants } \\
\text { not possible. } \\
\text { Investigators } \\
\text { blinded }\end{array}$ & $\begin{array}{l}\text { Low - Investigators, } \\
\text { study statistician } \\
\text { and data managers } \\
\text { were blinded } \\
\text { throughout. }\end{array}$ & $\begin{array}{l}\text { Unclear - Not } \\
\text { all outcome data } \\
\text { was reported } \\
(6 \mathrm{MWT})\end{array}$ & $\begin{array}{l}\text { Unclear - No } \\
\text { reporting of } \\
\text { one variable } \\
(6 \mathrm{MWT})\end{array}$ & $\begin{array}{l}\text { Details regarding } \\
\text { Wii intervention } \\
\text { are vague and } \\
\text { lack detail }\end{array}$ \\
\hline Yoon, et al. [20] & $\begin{array}{l}\text { Unclear - Stratified } \\
\text { random sampling } \\
\text { used }\end{array}$ & $\begin{array}{l}\text { Unclear - } \\
\text { Concealment } \\
\text { unspecified }\end{array}$ & $\begin{array}{l}\text { Unclear-Blinding } \\
\text { of participants } \\
\text { not specified }\end{array}$ & $\begin{array}{l}\text { Low - Evaluation } \\
\text { performed by a } \\
\text { blinded occupation } \\
\text { therapist }\end{array}$ & $\begin{array}{l}\text { Low - No } \\
\text { missing outcome } \\
\text { data. }\end{array}$ & $\begin{array}{l}\text { Low - All } \\
\text { outcomes } \\
\text { reported } \\
\text { and discussed } \\
\text { sufficiently. }\end{array}$ & \\
\hline
\end{tabular}


considered by the authors, with only four from 10 rated as 'Can't tell' (Table 2).

The remaining four studies were assessed using the Quality Checklist for Healthcare Intervention Studies (Table 3). Of these four studies, three were feasibility interventions [44, 45, 47, 48], with the final study a single-subject case study [49]. The three feasibility studies showed relatively good study quality with the lowest score being 20/30, whilst the case study was rated relatively low at 11/30 [49].

\section{Study populations}

The average number of participants with cancer who completed the studies was $12 \pm 12$, ranging from one, in the case-study [49], to 40 . The average age of the participants was $57 \pm 17$ years, with a range of $20-75$ years, with both males $(55 \%)$ and females (45\%) included. The diagnosis of participants included breast, lung, brain, rectal, oesophageal, tongue and prostate cancer, as well as leukemia and lymphoma. The severity and stage of cancer varied. The sole participant in the study from Betker, et al. [49] had a cerebella tumour with severe ataxia, whilst those who partook in the intervention by Hoffman, et al. [44, 45] all had lung cancer, ranging from stage $1 \mathrm{~A}$ to $3 \mathrm{~A}$ (Table 4).

\section{Study interventions}

Four of the included interventions implemented the Nintendo Wii for their exergaming intervention [26, 44$46,48]$. The remaining studies used a custom made centre of pressure (COP) video game combined with a pressure mat for interaction [49], a BrightArm Duo Rehabilitation System [47] or the Interactive Rehabilitation and Exercise (IREX) system [20]. Session duration ranged from 20 to
$50 \mathrm{~min}$; however, not all durations were reported, as this was sometimes determined by the patients. Frequency of the sessions ranged from two days per week to at least five days per week. Again this was unclear as to the maximum frequency the intervention was carried out as it depended upon each individual's confidence and motivation to persist. The duration of the interventions was on average 6.3 weeks, ranging from five days to 16 weeks. Only two of the studies included a comparison group [20, 46]. The study by Sajid, et al. [46] included an exergaming group (Wii), an exercise group (EXCAP) and a usual care group. The study by Yoon, et al. [20] included an exergaming group (IREX) combined with conventional occupational therapy (COT) and a COT only group (Table 5).

The interventions were delivered at various times, with one [44,45] collecting baseline measures prior to surgery, post-surgery, and each week for 16 weeks. Two studies were implemented on patients with a current cancer diagnosis receiving treatment $[26,48]$, with one study taking place 9.5 years post-surgery [47]. The study by Sajid, et al. [46] took place 62 month after androgen deprivation therapy (ADT) initiation, whilst Yoon, et al. [20] assessed participants nine month after diagnosis. Three interventions were carried out within a hospital setting $[26,48,49]$, two in the participants' home [44-46] and one in a clinical laboratory [47] (Table 4). The interventions were delivered by a physiotherapist [48, 49], an occupational therapist [20, 47], nurse [44, 45], research staff [26] or an exercise physiologist [46] (Table 5).

\section{Feasibility and acceptability of an exergaming intervention} Feasibility of exergaming interventions was assessed through retention rates and adherence rates whilst acceptability was

Table 2 Study quality assessment for qualitative studies [26] using the CASP Tool

\begin{tabular}{|c|c|c|c|}
\hline & Yes No & Can't tell & Comments \\
\hline $\begin{array}{l}\text { 1) Was there a clear statement of the aims } \\
\text { of the research? }\end{array}$ & $\checkmark$ & & Aim and rationale of the study is clear. \\
\hline 2) Is a qualitative methodology appropriate? & $\checkmark$ & & Methodology necessary in order to evaluate participants' experiences. \\
\hline $\begin{array}{l}\text { 3) Was the research design appropriate to } \\
\text { address the aims of the research? }\end{array}$ & $\checkmark$ & & Research design explained and justified in accordance with research goals. \\
\hline $\begin{array}{l}\text { 4) Was the recruitment strategy appropriate } \\
\text { to the aims of the research? }\end{array}$ & $\checkmark$ & & Clear explanation as to how participants were recruited and justified sample size \\
\hline $\begin{array}{l}\text { 5) Was the data collected in a way that } \\
\text { addressed the research issue? }\end{array}$ & & $\checkmark$ & $\begin{array}{l}\text { Data collection setting and data collection method wasn't justified. Detail as to } \\
\text { how interviews were recorded and transcribed. }\end{array}$ \\
\hline $\begin{array}{l}\text { 6) Has the relationship between researcher } \\
\text { and participants been adequately considered? }\end{array}$ & $\checkmark$ & & Researcher informs of no relationship with the participants. \\
\hline $\begin{array}{l}\text { 7) Have ethical issues been taken into } \\
\text { consideration? }\end{array}$ & & $\checkmark$ & $\begin{array}{l}\text { Brief explanation of how participants were informed of the research and no } \\
\text { detail on maintenance of ethical standards. }\end{array}$ \\
\hline 8) Was the data analysis sufficiently rigorous? & & $\checkmark$ & Brief detail of data analysis. Semi-structured interview could be source of bias. \\
\hline 9) Is there a clear statement of findings? & & $\checkmark$ & $\begin{array}{l}\text { Research hasn't discussed credibility of their findings. No information regarding } \\
\text { second analyst for transcriptions and interpretations. }\end{array}$ \\
\hline 10) How valuable is the research? & $\checkmark$ & & $\begin{array}{l}\text { Research contributes to existing knowledge and identifies new areas to be } \\
\text { researched }\end{array}$ \\
\hline
\end{tabular}




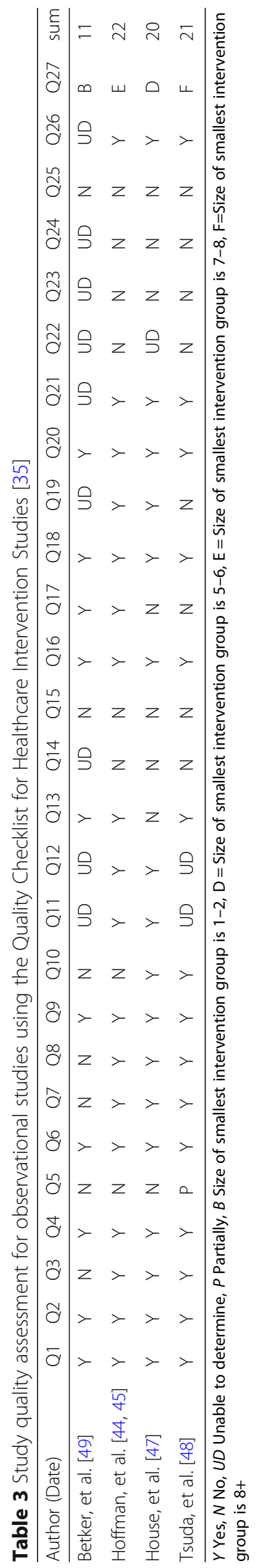




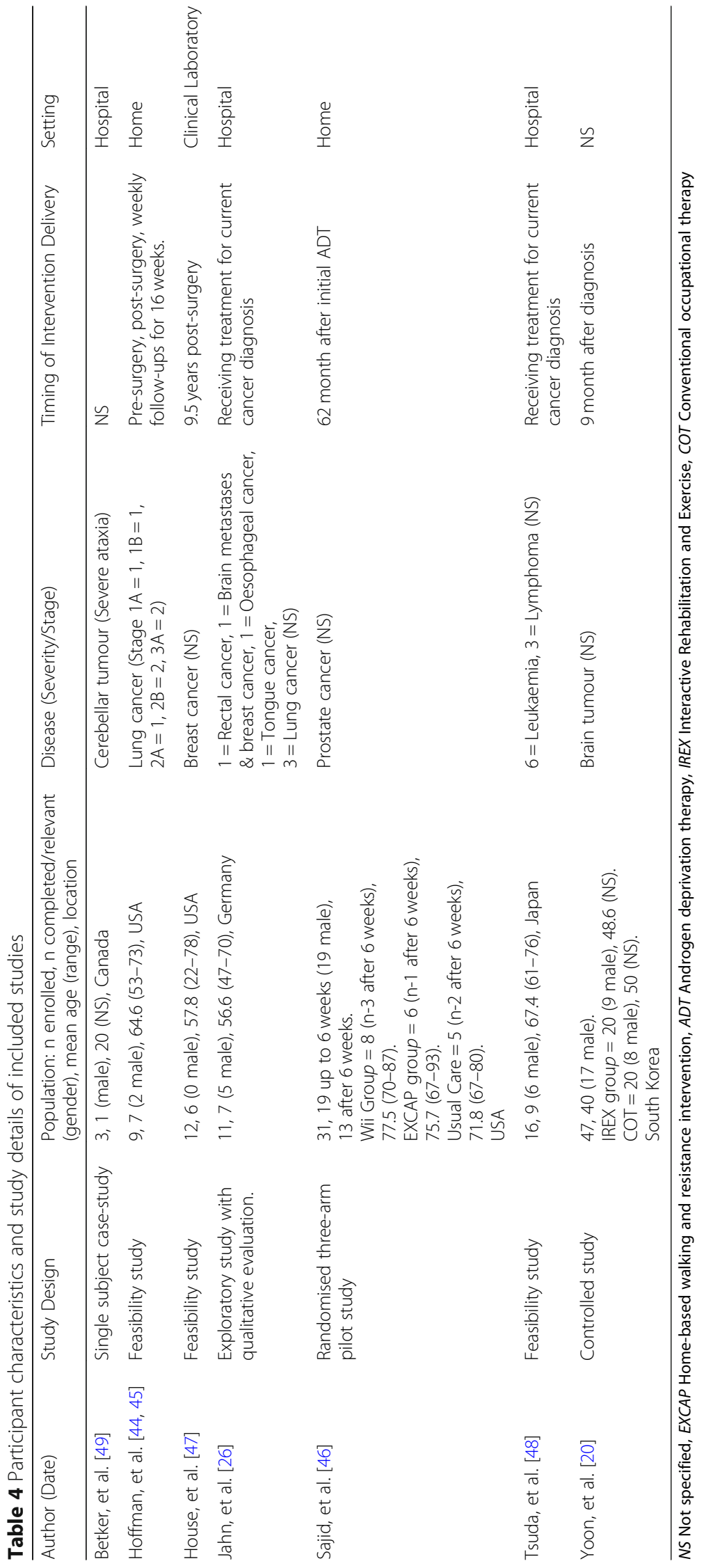




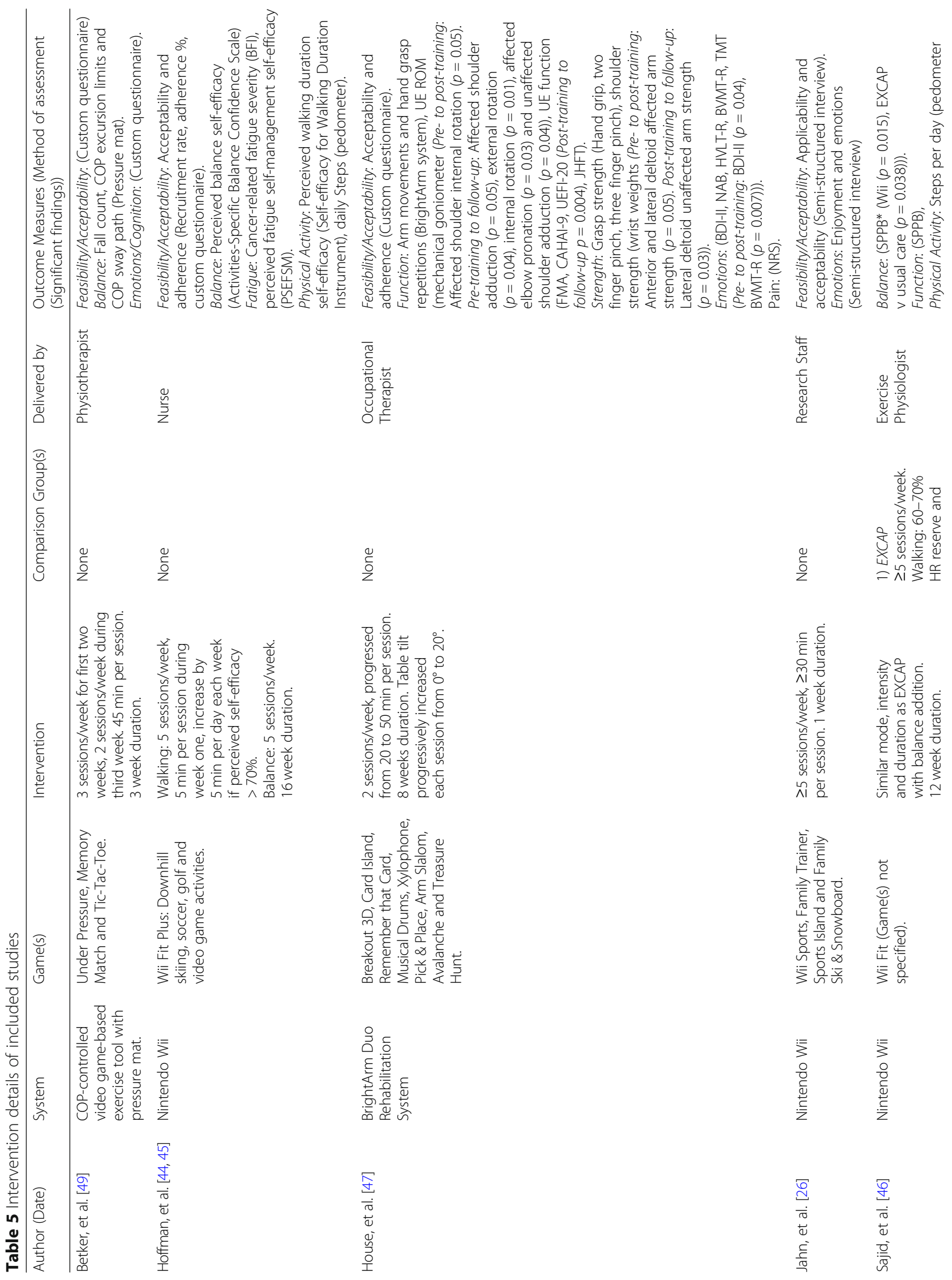




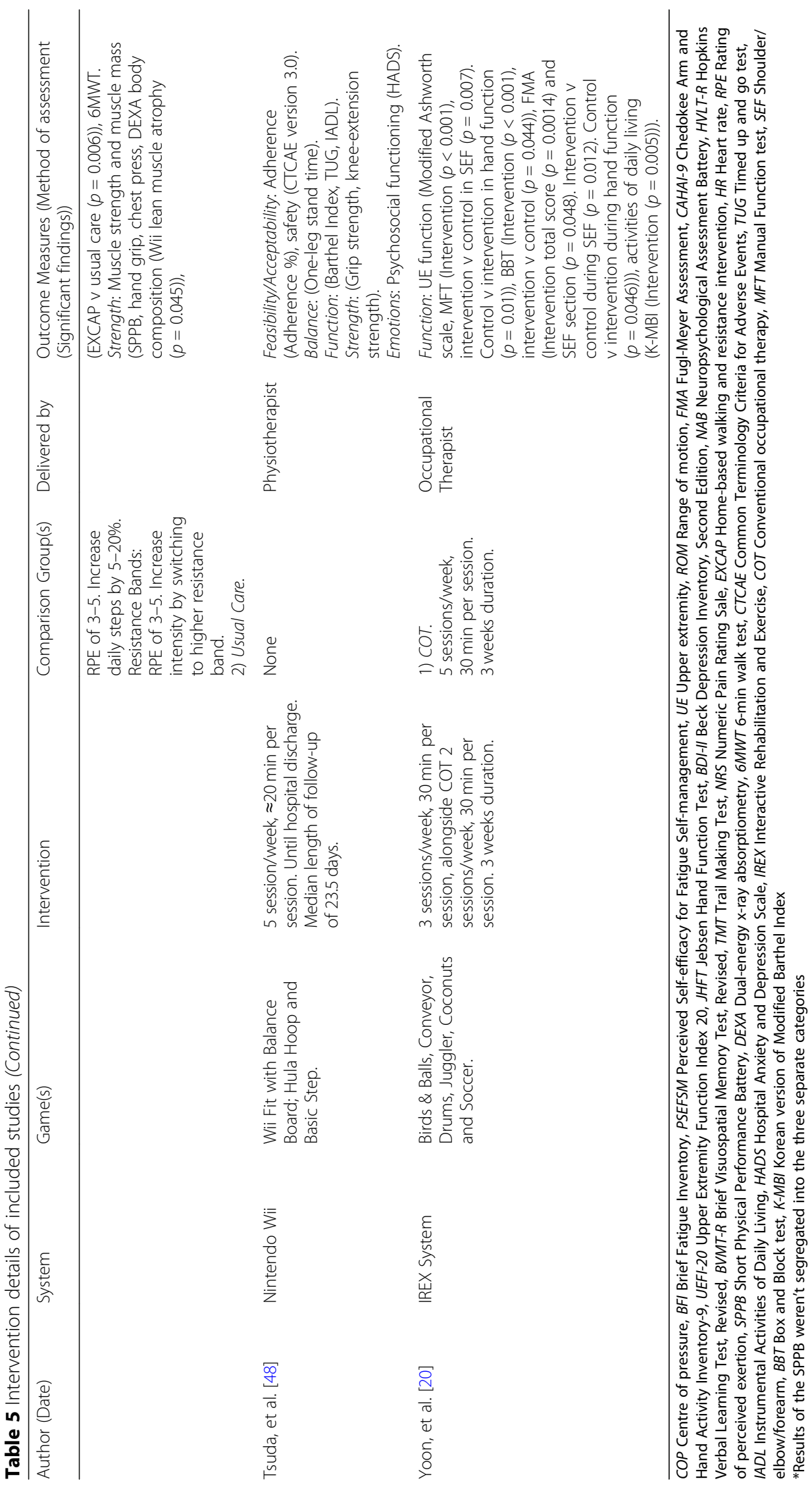


assessed through custom acceptability questionnaires and interviews.

Five studies assessed the feasibility and acceptability of using exergaming for cancer rehabilitation, determined through adherence percentage $[44,45,47,48]$, retention rate $[44,45,48]$, a custom evaluation questionnaire $[44,45,47,49]$ and a semi-structured interview [26].

The study by Hoffman, et al. [44, 45] reported an adherence rate of $96.6 \%$ after six weeks, which fell to $87.6 \%$ after 16 weeks. All participants within this study continued throughout the 16 weeks, giving a retention rate of $100 \%$. The participants in the study by Tsuda, et al. [48] had an adherence rate of $62 \%$ and a retention rate of $56 \%$; however, only one dropout wasn't due to ill health. Any sessions missed by those in the study by House, et al. [47] were made up during alternative days, leading to a $100 \%$ adherence rate. Of the 12 participants enrolled, only six completed the testing, leading to a $50 \%$ retention rate.

Only one study assessed the safety of the exergaming intervention using a set of criteria to grade any adverse effects. Tsuda, et al. [48] found there to be no musculoskeletal or adverse events associated with the intervention.

The studies that implemented a custom evaluation questionnaire showed high acceptability of 5.8/6 [44, 45] and 3.9/5 after four weeks increasing to $4.5 / 5$ after eight weeks, with higher scores indicative of a high level of acceptance [47]. The questionnaire used by Betker, et al. [49] only included three questions; however, one of these showed that the exergaming intervention was preferred to exercise programs the participant had previously partaken in. When participants were asked for their opinion of the exergaming interventions, they were described as fun, easy to use, convenient, comfortable, relaxing and would recommend their use [26, 44, 45, 47].

\section{The outcomes of exergaming interventions}

Results of the exergaming interventions were assessed through many measures. These included balance, function, physical activity, strength, fatigue, emotions, cognition and pain.

\section{Physical functioning}

Betker, et al. [49] assessed pre- and post-intervention balance and falls during numerous $20 \mathrm{~s}$ tests, such as eyes open, eyes closed, unipedal, bipedal and differing surfaces, along with COP measurements. The sole participant in this study improved their fall count (unable to maintain balance) from 10 falls from 12 tests pre-intervention to one fall from the same tests post-intervention, with an improved COP range during all tests.

One study assessed participants' perceived balance confidence, using the Activities-Specific Balance Confidence Scale [44, 45]. Pre-surgery scores were $86 \%$, falling to $72.8 \%$ post-surgery. At post-intervention, the perceived balance confidence scores increased to $83.7 \%$ and then to $88.9 \%$ after week six and 16 , respectively. The average length of hospital stay was eight days, with the intervention beginning on average $32 \mathrm{~h}$ following discharge.

The Short Physical Performance Battery (SPPB) was used in one study [46], which includes a balance component. The results of this, however, weren't segregated from the other components of this test. The final study to assess balance used a baseline and post-exercise one leg standing time [48], finding no significant improvement (left leg $p=1$; right leg $p=0.1$ ).

House, et al. [47] assessed function through arm movements, hand grasp repetitions, upper extremity (UE) ROM, Fugl-Meyer Assessment (FMA), Chedokee Arm and Hand Activity Inventory-9 (CAHAI-9), Upper Extremity Function Index 20 (UEFI-20) and Jebsen Hand Function Test (JHFT). Arm movements and hand grasp repetitions steadily increased from session one through to 16 . ROM increased among almost all movements assessed, with only a decrease in unaffected shoulder external rotation. Significant improvements were seen in the affected shoulder internal rotation $(p=0.05)$ between pre- and post-training, the affected shoulder adduction $(p=0.05)$, internal rotation $(p=0.01)$ and external rotation $(p=0.04)$ and the affected elbow pronation $(p=0.03)$ between pre-training and the eight week follow-up post-intervention. Improvements were also seen in the other aforementioned tests, barring the JHFT in the unaffected shoulder which decreased. UEFI-20 was significantly greater at the eight week follow up compared to pre-training $(p=0.004)$.

Function was partly measured through the implementation of the SPPB in the study by Sajid, et al. [46] which includes the 4-m gait speed test. Again, these results weren't isolated from the other components of this test. The Barthel Index, Timed Up and Go (TUG) test and the Instrumental Activities of Daily Living scale (IADL) were used in another study [48]. No difference was seen between baseline and post-exercise measures for the Barthel Index; however, the TUG showed a decrease $(p=0.58)$, with slight improvements seen during the IADL $(p=1)$.

The Modified Ashworth scale, Manual Function Test (MFT), Box and Block Test (BBT), FMA and the Korean version of the Modified Barthel Index (K-MBI) was used in the study by Yoon, et al. [20]. Significant improvements in functional dexterity were found through the BBT $(p=0.044)$ in the combined exergaming and COT group compared to the COT group alone. It was also found that shoulder/elbow/forearm (SEF) function improved significantly among the combined exergaming group during the MFT $(p=0.007)$ and FMA $(p=0.012)$ in comparison to the COT group. Hand function improved significantly within the control group in comparison to the combined exergaming and COP group $(p=0.01, p=0.046)$ during the MFT and FMA, respectively. 
A pedometer was used to assess daily steps completed, whilst the Self-efficacy for Walking Duration Instrument was used to assess perceived walking self-efficacy among participants in the study by Hoffman, et al. [44, 45]. Steps per day increased from 4650 during week one, to 6393 during week six, before fluctuating on a weekly basis thereafter. Perceived self-efficacy for walking duration was based on participant's perception of being able to walk for $30 \mathrm{~min}$. This self-efficacy dropped from $96.4 \%$ pre-surgery to $47.4 \%$ post-surgery, before increasing to $99.4 \%$ at the end of the intervention at 16 weeks.

A pedometer was also used to measure steps taken per day in another study [46]. The results showed an increased in all three groups, with the exercise group significantly greater than the usual care group $(p=0.006)$.

House, et al. [47] assessed UE strength through a hand grip test, a two and a three finger pinch test as well as wrist weights used to measure shoulder strength, all of which improved in the affected shoulder from pre- to post-training and again after the eight week follow-up. Anterior and lateral deltoid strength significantly improved between pre- and post-training $(p=0.05)$ following the intervention. A similar trend was also seen for the unaffected arm strength, despite the two fingers pinch score decreasing. Lateral deltoid strength of the unaffected shoulder increased significantly between pre-training and eight week follow-up $(p=0.03)$.

Chest press, handgrip dynamometer test and a dual energy $\mathrm{x}$-ray absorptiometry (DEXA) scan was completed to assess muscle mass and muscle strength in another study [46]. Handgrip scores and lean muscle mass $(p=0.045)$ decreased in the Wii group, whilst the EXCAP group seen an increase in handgrip strength, with a decrease in lean muscle mass, both of which non-significant.

The final study to assess strength [48] used grip strength and knee extension strength. Knee extension strength increased non-significantly post-intervention, in comparison to baseline measures. Grip strength increased in the left hand, with a decrease in the right hand, both of which non-significant.

\section{Symptoms}

Only one intervention [44, 45] assessed fatigue. This was done using the Brief Fatigue Inventory (BFI) and also assessed participants' perceived self-efficacy for managing fatigue using the Perceived Self-efficacy for Fatigue Self-management (PSEFSM) instrument. On a scale of 010 , with 10 being 'most severe', the scores of the BFI reveal a pre-surgery score of 3.3 , rising to 4.8 post-surgery. This value fell to 2.8 after week six, and again to 1.32 at week 16 . Perceived self-efficacy for managing fatigue rose from 7.0 pre-surgery to 7.1 post-surgery before falling until week two at 5.4, before rising to 7.7 and remaining between 7 and 9 for the remainder of the 16 week intervention.
The participant in the study by Betker, et al. [49] said they had fun partaking in the exergaming intervention. They stated they experienced a loss of time awareness, as found through a short custom questionnaire.

The study by House, et al. [47] used the Beck Depression Inventory second edition (BDI-II), the Neuropsychological Assessment Battery (NAB), Hopkins Verbal Learning Test, Revised (HVLT-R), the Brief Visuospatial Memory Test, Revised (BVMT-R) and the Trail Making Test (TMT) A and $\mathrm{B}$. Improvements were seen between pre- and post-training in all tests, with the BDI-II and BVMT-R improving significantly ( $p=0.04$ and $p=0.007$, respectively). No change was seen in the 'Person' category in the NAB, and the TMT-A showed a non-significant decrease. No significant improvements were seen between pre-training and at eight week follow-up, despite improvements in eight out of 12 tests. Tsuda, et al. [48] used the Hospital Anxiety and Depression Scale (HADS) to measure the psychosocial functioning of participants. Both anxiety and depression decreased within this study, with anxiety scores approaching significance $(p=0.055)$.

Interviews were carried out in the study by Jahn, et al. [26]. Through these interviews, participants responded saying they felt a decrease in negative emotions such as stress and that they felt more relaxed and had an increased mood state following the intervention.

Pain was only assessed in one study, using the Numeric Pain Rating Scale (NRS) [47]. Pain showed a slight decrease, with the worst pain reported in week one as $5 / 10$, with a decrease of 1.1 over the eight week period $(p=0.1)$.

\section{Discussion}

This is the first systematic review to synthesise current evidence in order to describe exergaming interventions delivered to individuals with a current or previous diagnosis of cancer and to explore the feasibility, acceptability and outcomes of such interventions. The interventions found through this review showed great variability to one another.

Due to the variability of the interventions included, it is difficult to conclude which method of delivery would prove most advantageous. No one mode of intervention appeared to be most beneficial, although the majority used the Nintendo Wii. Almost half of the interventions were delivered within the hospital, with two delivered at home, indicating the suitability of exergaming interventions within both environments. Interventions showing significant improvements in health outcomes were delivered by an occupational therapist. The only other intervention to report an improvement was delivered by an exercise physiologist. It would appear exergaming interventions are most successful when delivered by a qualified healthcare professional or a professional with knowledge of exercise prescription. Duration and frequency of interventions varied greatly, however longer and more frequent interventions 
didn't incur greater improvements. Balance, function, emotions and fatigue were the health outcomes most frequently assessed and targeted by exergaming interventions.

Exergaming interventions appear to be feasible and acceptable to individuals with cancer. It is difficult, however, to draw firm conclusions regarding its benefits due to the heterogeneity in the delivery of interventions and the outcome measures assessed within the included studies.

Adherence to interventions delivered to individuals with cancer and consisting of exercise, is relatively low $[16,18,21]$ potentially due to the monotonous nature of aerobic and resistance training [50]. Numerous other factors have been shown to be associated with adherence rates among cancer patients, including, but not limited to, exercise history, fatigue levels, body mass index and level of education [51, 52]. Adherence percentages within this review $[44,45,47,48]$ were positive, with the lowest being $62 \%$; however only one drop-out in this study wasn't due to ill health, displaying high feasibility among those who are well, but also highlighting a challenge of delivering exercise interventions to a vulnerable group of individuals. Only one study within this review assessed the safety of the intervention using a set of criteria to grade any adverse events. No musculoskeletal or adverse events occurred [48].

Exergaming interventions have been shown to be acceptable to older adults [53], and those with chronic conditions, including PD [24], MS [54], chronic obstructive pulmonary disease (COPD) [55] and stroke [56] patients, concurring with results from the current review. Older adults [53], PD [24] and post-stroke patients [56] found the interventions to be fun and enjoyable, whilst older adults [53], PD [24] and MS patients [54] were motivated to participate. MS patients [54] showed greater intrinsic motivation through the Flow State Scale than those undertaking traditional training. Post-stroke patients [56] also found the exergaming intervention to be useful, if not better, than conventional rehabilitation, and would recommend its use to other patients. Conversely, COPD patients [55] accepted a pulmonary rehabilitation program (PRP) more than the exergaming intervention, albeit non-significantly. Barry, et al. [24] found that fast-paced and complex games caused difficulties and that games should be tailored according to the needs of the clinical population.

Exergaming has already been shown to be an effective method to increase physical activity in chronic disease populations, including PD [24], MS [31], cystic fibrosis [32] and those who have recently suffered a stroke [33], with little research assessing exergaming in a cancer population. Through this review, exergaming appears to improve several health aspects, such as balance, function, physical activity, strength, fatigue, emotions, cognition and pain among cancer patients.

Balance was shown to improve through two interventions within this review [44, 45, 49]. Fall counts and postural sway decreased in one study [49], whilst Hoffman, et al. [44, 45] found that participants' balance self-efficacy increased. Exergaming has also been shown to improve balance among older adults $[27,53]$ and clinical populations, including PD [24], MS [54, 57] and post-stroke [33] patients. A study among older participants found a Wii intervention to significantly improve results of the Berg Balance Scale, between baseline and week four compared to standard care alone [27].

Function was assessed in four interventions [20, 46-48] within this review, and was shown to improve, whether this was improved arm movements, hand grasp repetitions or functional dexterity. Exergaming interventions have been shown to have mixed results on functionality among older adults and clinical populations. Among older participants, dual-task function has been shown to improve in some studies, following a systematic review; however, many significant findings weren't in comparison to a control group [34]. PD patients showed an increase in functionality following a variety in exergaming intervention, through a variety of tests as shown through a systematic review [24], whilst MS patients displayed mixed results, with non-significant improvements in the TUG and $25 \mathrm{ft}$ walk test, and significant improvements for both the exergaming and control group during the Dynamic Gait Index [31]. PD patients' function has shown to improve, which coincides with the findings from the current review. MS and older participants' function meanwhile is not as conclusive.

Physical activity was assessed within two interventions in this review [44-46]. Steps per day were shown to increase among those who partook in an exergaming intervention within this review; however, not as much as a standard exercise group. Although little research exists in exergaming interventions, standard exercise interventions have shown improvements in exercise capacity among COPD patients, potentially allowing for an improvement in physical activity [58, 59].

Exergaming interventions were shown to improve several measures of strength, assessed during three interventions [46-48], throughout this review. One study found that despite improvements, a standard exercise group improved more than the exergaming group [46]. PD patients have shown significant improvements in their strength following an exergaming intervention [60]. These improvements were seen for both the exercise and exergaming group within the study, with no significant difference between the two. Post-stroke patients saw a significant improvement of strength following an exergaming intervention, whilst recreation therapy showed a non-significant increase [61]. This previous literature coincides with the findings from the current review. Despite improvements, it is inconclusive as to whether exergaming is more beneficial than a standard exercise intervention. 
Exergaming was seen to decrease levels of fatigue in the one intervention $[44,45]$ where it was assessed through the current review. This finding concurs with findings of exergaming interventions among PD [62], MS [63] and COPD [64] patients, along with those who were undergoing a PRP [65].

Four interventions [26, 47-49] in this systematic review found that exergaming had a positive impact on the emotions and cognition of cancer patients. Depression, mood and relaxation all improved following the interventions. Older participants have shown an increase in emotions and cognition following an exergaming intervention. A scoping review carried out by Klompstra, et al. [53] found that three studies reported an increase in cognitive function [66-68], with one showing a decrease in depressive symptoms [68]. Likewise, PD [69] patients have also shown to have an improved mood, whilst COPD [55] patients have shown a significant decrease in depressive symptoms and a non-significant decrease in anxiety among the exergaming group. Changes were however less than the PRP alone, which seen significant decreases among both outcomes. The findings of the current review coincide with this previous literature.

Pain was only assessed in one intervention in this review [47], showing a slight decrease following an exergaming intervention. These findings are in keeping with other studies which have implemented exergaming, conducted in post-stroke patients [56], those having undergone a knee replacement [70] and also with UE dysfunction [71]. A systematic review also revealed that four of seven studies found significant improvements in musculoskeletal pain following exergaming interventions [72].

This systematic review is not without its limitations. The studies which were included within this review were vastly different from one another in terms of the console used, game played, the diagnosis of cancer and the outcomes assessed, which made analyses of the effects of exergaming difficult to derive, and a meta-analyses unable to be performed. Therefore no conclusion could be made on a single exergame or specific console, rather exergaming generically as a whole. Due to the relatively small number of studies included, conclusions drawn are tentative, however this review offers important insights regarding the delivery of exergaming to individuals with cancer. This review only found one study which assessed the safety of the intervention which is an important factor in determining feasibility. The safety of interventions should be considered in future studies applying exergaming interventions in a cancer population by reporting adverse events using set criteria. Other threats to internal and external validity exist within the included studies. Only one of the studies included was a randomised control trial thus conclusions on the outcomes of exergaming are taken with caution. Reviews with few or no randomised controlled trials are still valuable for inclusion within systematic reviews as they provide information regarding the feasibility of an intervention [73]. They can also prove to be of high value and of high interest to patients, healthcare professionals and stakeholders $[74,75]$. The sample sizes within the studies were also relatively small, in one case with only one eligible participant. Due to this, it is difficult to draw firm conclusions from the results of the analyses due to the limited statistical power. The criteria of any cancer patients meant that a variety of cancers were researched, meaning no conclusion could be made on a specific diagnosis or disease severity. The lack of control group in many of the studies meant that it was difficult to determine whether the exergaming interventions were the cause of improvement, or whether these would have been shown following a standard exercise based regime. The inclusion criterion for this review was very broad which allowed for a range of studies to be included. In doing so, it provides a general insight into how exergaming has been used for patients with a current or previous diagnosis of cancer.

It is clear, through this review, that due to the scarcity of literature within this field, more research is required. Future research should explore common outcome variables during exergaming interventions, suitable to all cancer groups. It is also recommended that a control group, using standard exercise therapy, should be included in order to determine whether improvements are solely due to the exergaming intervention. Studies should also look to implement longer follow-ups in order to assess the feasibility, acceptability and outcomes of an exergaming intervention over a prolonged period of time.

\section{Conclusion}

In conclusion, the content of the interventions delivered to cancer patients show great heterogeneity, along with a wide variety of health outcomes assessed, therefore it is difficult to conclude its effectiveness. However, adherence rates and enjoyment appear greater during exergaming interventions compared to standard care, highlighting the feasibility and acceptability of this type of intervention delivery for adults with cancer.

\section{Appendix}

Search Strategy.

(exergam* or activ* n3 video n3 gam* or activ*n3 videogam* or human $n 3$ computer $n 3$ interaction or virtual $n 3$ reality or virtual $n 3$ world or augment* $n 3$ reality or mobile $\mathrm{n} 3 \mathrm{app}^{*}$ or exert* $\mathrm{n} 3$ interfac* or electronic $\mathrm{n} 3$ gam* $^{*}$ or activ* n3 gam*n3 play or virtual n3 rehab* or augment* $\mathrm{n} 3$ rehab* $^{*}$ or mHealth or Wii or Eye $\mathrm{n} 3$ Toy or Eyetoy or Kinect or DDR or Dance n3 Dance n3 Revolution or IREX) and (neoplasm* or cancer* or carcino* $^{*}$ or leukemia or leukaemia or tumor or tumour or NSCLC or non n3 small n3 cell or thorac* or postthorac*). 


\section{Abbreviations}

6MWT: 6-minutemin walk test; ADT: Androgen deprivation therapy; BBT: Box and Block Test; BDI-II: Beck Depression Inventory second edition; BFI: Brief Fatique Inventory; BVMT-R: Brief Visuospatial Memory Test, Revised; CAHAI9: Chedokee Arm and Hand Activity Inventory-9; CASP: Critical Appraisal Skills Program; COP: Centre of pressure; COPD: Chronic obstructive pulmonary disease; COT: Conventional occupational therapy; CRF: Cancer-related fatigue; CTCAE: Common Terminology Criteria for Adverse Events; DEXA: Dual energy x-ray absorptiometry; FMA: Fugl-Meyer Assessment; HADS: Hospital Anxiety and Depression Scale; HR: Heart rate; HVLT-R: Hopkins Verbal Learning Test, Revised; IADL: Instrumental Activities of Daily Living; IREX: Interactive Rehabilitation and Exercise; JHFT: Jebsen Hand Function Test; K-MBI: Korean version of the Modified Barthel Index; MFT: Manual Function Test: MS: Multiple sclerosis; NAB: Neuropsychological Assessment Battery; NRS: Numeric Pain Rating Scale; PD: Parkinson's disease; PRP: Pulmonary rehabilitation program; PSEFSM: Perceived Self-efficacy for Fatigue Selfmanagement; QOL: Quality of life; ROM: Range of motion; RPE: Rate of perceived exertion; SEF: Shoulder/elbow/forearm; SPPB: Short Physical Performance Battery; TMT: Trail Making Test; TUG: Timed Up and Go; UE: Upper extremity; UEFI-20: Upper Extremity Function Index 20

\section{Acknowledgements}

Nicola Conway: Assisted with the designing of the search strategies in collaboration with the main researcher (DT).

\section{Funding}

No funding was obtained for this project.

\section{Availability of data and materials}

Not applicable.

\section{Authors' contributions}

DT designed the search strategy in conjunction with a professional librarian, searched the literature, removed duplicated studies, removed irrelevant articles, extracted and interpreted the data, completed study quality assessments and wrote the manuscript. SG checked half of the search results, removed irrelevant articles, checked extracted data and completed study quality assessments. PR checked the remaining half of the search results, removing irrelevant articles. The remaining authors (SH, JD, JR) conceptualised the review ideas alongside DT and supervised the project. All authors approved the final version of the manuscript.

\section{Ethics approval}

Not applicable.

\section{Consent for publication}

Not applicable.

\section{Competing interests}

The authors declare that they have no competing interests.

\section{Publisher's Note}

Springer Nature remains neutral with regard to jurisdictional claims in published maps and institutional affiliations.

\section{Author details \\ ${ }^{1}$ School of Health and Social Care, Teesside University, Middlesbrough TS1 3BX, UK. ${ }^{2}$ Department of Sport and Exercise Sciences, University of Sunderland, Sunderland SR1 3SD, UK. \\ Received: 9 January 2018 Accepted: 8 November 2018 Published online: 21 November 2018}

\section{References}

1. World Health Organisation (WHO), "Cancer," 2017. [Online]. Available: http:// www.who.int/news-room/fact-sheets/detail/cancer. [Accessed 16 Mar 2017].

2. Kochanek K, Murphy S, Xu J, Tejada-Vera B. Deaths: final data for 2014. National Vital Statistic Reports. 2016;65(4):1-122.

3. Barsevick A. The elusive concept of the symptom cluster. Oncol Nurs Forum. 2007;34(5):971-80.
4. Cheng K, Lee D. Effects of pain, fatigue, insomnia, and mood disturbance on functional status and quality of life of elderly patients with cancer. Crit Rev Oncol Hematol. 2011;78(2):127-37.

5. Theobald D. Cancer pain, fatigue, distress, and insomnia in cancer patients. Clinical Cornerstone. 2004;6(1D):S15-21.

6. Gritsenko V, Dailey E, Kyle N, Taylor M, Whittacre S, Swisher A. Feasibility of Using Low-Cost Motion Capture for Automated Screening of Shoulder Motion Limitation after Breast Cancer Surgery. PLoS One. 2015;10(6):e0128809.

7. Van Gerpen R, Becker B. Development of an evidence-based exercise and education cancer recovery program. Clin J Oncol Nurs. 2013;17(5):539-43.

8. K. Schmitz, K. Courneya, C. Matthews, W. Demark-Wahnefried, D. Galvão, B. Pinto, M. Irwin, K. Wolin, R. Segal, A. Lucia, C. Schneider, V. von Gruenigen, A. Schwartz and A. C. o. S. Medicine, "American College of Sports Medicine roundtable on exercise guidelines for cancer survivors," Med Sci Sports Exerc, vol. 42, no. 7, pp. 1409-1426, 2010

9. National Comprehensive Cancer Network (NCCN), "Clinical Practice Guidelines in Oncology (NCCN Guidelines ${ }^{\circledR}$ : Cancer-Related Fatigue, Version 2," 2017. [Online]. Available: https://www.nccn.org/professionals/physician_ gls/PDF/fatigue.pdf. [Accessed 1 May 2017].

10. Kenfield S, Stampfer M, Giovannucci E, Chan J. Physical activity and survival after prostate cancer diagnosis in the health professionals follow-up study. J Clin Oncol. 2011;29(6):726-32.

11. Lee J. A meta-analysis of the association between physical activity and breast Cancer mortality. Cancer Nurs. 2018.

12. Schmid D, Matthews CE, Leitzmann MF. Physical activity and sedentary behavior in relation to mortality among renal cell cancer survivors. PLoS One. 2018;13(6):e0198995.

13. Meyerhardt J, Heseltine D, Niedzwiecki D, Hollis D, Saltz L, Mayer R, Thomas J, Nelson H, Whittom R, Hantel A, Schilsky R, Fuchs C. Impact of physical activity on cancer recurrence and survival in patients with stage III colon cancer: findings from CALGB 89803. J Clin Oncol. 2006;24(22):3535-41.

14. Bourke L, Homer K, Thaha M, Steed L, Rosario D. Interventions to improve exercise behaviour in sedentary people living with and beyond cancer: a systematic review. Br J Cancer. 2014;110(4):831-41.

15. Cramp F, Byron-Daniel J. Exercise for the management of cancer-related fatigue in adults. Cochrane Database of Syst Rev. 2012;11:CD006145.

16. Velthuis M, Agasi-Idenburg S, Aufdemkampe G, Wittink $H$. The effect of physical exercise on cancer-related fatigue during cancer treatment: a metaanalysis of randomised controlled trials. Clin Oncol. 2010;22(3):208-21.

17. Kangas M, Bovbjerg D, Montgomery G. Cancer-related fatigue: a systematic and meta-analytic review of non-pharmacological therapies for cancer patients. Psychol Bull. 2008;134(5):700-41.

18. Alibhai S, O'Neill S, Fisher-Schlombs K. A clinical trial of supervised exercise for adult inpatients with acute myeloid leukemia (AML) undergoing induction chemotherapy. Leuk Res. 2012;36(10):1255-61.

19. Swisher A, Erickson M. Perceptions of physical activity in a group of adolescents with cystic fibrosis. Cardiopulmonary Physical Therapy Journal. 2008;19(4):107-13.

20. Yoon J, Chun M, Lee S, Kim B. Effect of virtual reality-based rehabilitation on upper-extremity function in patients with brain tumor: controlled trial. Am J Phys Med Rehabil. 2015;94(6):449-59.

21. Quist M, Rørth M, Langer S, Jones L, Laursen J. Safety and feasibility of a combined exercise intervention for inoperable lung cancer patients undergoing chemotherapy: a pilot study. Lung Cancer. 2012;75(2):203-8.

22. Wardini R, Dajczman E, Yang N, Baltzan M, Préfontaine D, Stathatos M, Marciano $\mathrm{H}$, Watson S, Wolkove N. Using a virtual game system to innovate pulmonary rehabilitation: safety, adherence and enjoyment in severe chronic obstructive pulmonary disease. Can Respir J. 2013;20(5):357-61.

23. van Santen J, Dröes RM, Holstege M, Henkemans OB, van Rijn A, de Vries R, van Straten A, Meilanda F. Effects of exergaming in people with dementia: results of a systematic literature review. J Alzheimers Dis. 2018;63(2):741-60.

24. Barry $G$, Galna B, Rochester $L$. The role of exergaming in Parkinson's disease rehabilitation: a systematic review of the evidence. J Neuroeng Rehabil. 2014;11(33):33.

25. Barry G, Tough D, Sheerin P, Mattinson O, Dawe R, Board E. Assessing the physiological cost of active videogames (Xbox Kinect) versus sedentary videogames in young healthy males. Games for Health Journal. 2016;5(1):68-74.

26. Jahn P, Lakowa N, Landenberger M, Vordermark D, Stoll O. InterACTIV: an exploratory study of the use of a game console to promote physical activation of hospitalized adult patients with cancer. Oncol Nurs Forum. 2012;39(2):E84-90 
27. Williams M, Soiza R, Jenkinson A, Stewart A. EXercising with computers in later life (EXCELL) - pilot and feasibility study of the acceptability of the Nintendo ${ }^{\circledR}$ WiiFit in community-dwelling fallers. BMC Research Notes. 2010;3:238.

28. Skjæret N, Nawaz A, Morat T, Schoene D, Helbostad J, Vereijken B. Exercise and rehabilitation delivered through exergames in older adults: an integrative review of technologies, safety and efficacy. Int J Med Inform. 2016;85(1):1-16

29. Pompeu J, Arduini L, Botelho A, Fonseca M, Pompeu S, Torriani-Pasin C, Deutsch J. Feasibility, safety and outcomes of playing Kinect adventures! ${ }^{\mathrm{TM}}$ for people with Parkinson's disease: a pilot study. Physiotherapy. 2014;100(2):162-8.

30. Laver K, George S, Thomas S, Deutsch J, Crotty M. Virtual reality for stroke rehabilitation. Cochrane Database for Syst Reviews. 2011;9:CD008349.

31. Tariq $\mathrm{H}$. Effect of balance exercises for person with multiple sclerosis using Wii game: a systematic review of randomized and non-randomized control trials. Acta Medica International. 2016;3(1):196-201.

32. Carbonera R, Vendrusculo F, Donadio M. Physiological responses during exercise with video games in patients with cystic fibrosis: a systematic review. Respir Med. 2016;119:63-9.

33. Li Z, Han X, Sheng J, Ma S. Virtual reality for improving balance in patients after stroke: a systematic review and meta-analysis. Clin Rehabil. 2016;30(5): 432-40.

34. Ogawa E, You T, Leveille S. Potential benefits of exergaming for cognition and dual-task function in older adults: a systematic review. J Aging Phys Act. 2016;24(2):332-6.

35. Liberati A, Altman D, Tetzlaff J, Mulrow C, Gøtzsche P, loannidis J, Clarke M, Devereaux P, Kleijnen J, Moher D. The PRISMA statement for reporting systematic reviews and meta-analyses of studies that evaluate healthcare interventions: explanation and elaboration. J Clin Epidemiol. 2009;62(10):E1-E34.

36. Higgins JP, Altman D, Gøtzsche P, Jüni P, Moher D, Oxman A, Savovic J, Schulz K, Weeks L, Sterne J, C. B. M. Group and C. S. M. Group. The Cochrane Collaboration's tool for assessing risk of bias in randomised trials. BMJ. 2011;343:d5928.

37. Jørgensen L, Paludan-Müller AS, Laursen DRT, Savović J, Boutron I, Sterne JAC, Higgins JPT, Hróbjartsson A. Evaluation of the Cochrane tool for assessing risk of bias in randomized clinical trials: overview of published comments and analysis of user practice in Cochrane and non-Cochrane reviews. Syst Rev. 2016;5:80.

38. Downs S, Black N. The feasibility of creating a checklist for the assessment of the methodological quality both of randomised and non-randomised studies of health care interventions. J Epidemiol Community Health. 1998:52(6):377-84.

39. Deeks JJ, Dinnes J, D'Amico R, Sowden AJ, Sakarovitch C, Song F, Petticrew M, Altman DG. evaluating non-randomised intervention studies. Health Technol Assess. 2003;7(27):1-173.

40. Nye C, Hahs-Vaughn D. Assessing methodological quality of randomized and quasi-experimental trials: a summary of stuttering treatment research. Int J Speech Lang Pathol. 2011;13(1):49-60.

41. Critical Appraisal Skills Program (CASP), "CASP Checklists," 2013. [Online]. Available: http://media.wix.com/ugd/dded87_29c5b002d99342f788c6 ac670e49f274.pdf. [Accessed 28 Feb 2017].

42. Chenail RJ. Learning to appraise the quality of qualitative research articles: a contextualized learning object for constructing knowledge. Qual Rep. 2011;16(1):236-48

43. Nadelson S, Nadelson LS. Evidence-based practice article reviews using CASP tools: a method for teaching EBP. Worldviews Evid-Based Nurs. 2014;11(5):344-6.

44. Hoffman A, Brintnall R, Brown J, von Eye A, Jones L, Alderink G, Ritz-Holland $D$, Enter M, Patzelt L, VanOtteren G. Virtual reality bringing a new reality to postthoracotomy lung cancer patients via a home-based exercise intervention targeting fatigue while undergoing adjuvant treatment. Cancer Nurs. 2014;37(1):23-33.

45. Hoffman A, Brintnall R, Brown J, Eye A, Jones L, Alderink G, Ritz-Holland D, Enter M, Patzelt L, Vanotteren G. Too sick not to exercise: using a 6-week, home-based exercise intervention for cancer-related fatigue selfmanagement for postsurgical non-small cell lung cancer patients. Cancer Nurs. 2013;36(3):175-88.

46. Sajid S, Dale W, Mustian K, Kotwal A, Heckler C, Porto M, Fung C, Mohile S. Novel physical activity interventions for older patients with prostate cancer on hormone therapy: a pilot randomized study. J Geriatr Oncol. 2016;7(2): 71-80.
47. House G, Burdea G, Grampurohit N, Polistico K, Roll D, Damiani F, Hundal J, Demesmin D. A feasibility study to determine the benefits of upper extremity virtual rehabilitation therapy for coping with chronic pain postcancer surgery. Br J Pain. 2016;10(4):186-97.

48. Tsuda K, Sudo K, Goto G, Takai M, Itokawa T, Isshiki T, Takei N, Tanimoto T, Komatsu T. A feasibility study of virtual reality exercise in elderly patients with hematologic malignancies receiving chemotherapy. Intern Med. 2016;55(4):347-52

49. Betker A, Szturm T, Moussavi Z, Nett C. Video game-based exercises for balance rehabilitation: a single-subject design. Arch Phys Med Rehabil. 2006:87(8):1141-9.

50. Albiol-Pérez S, Forcano-García M, Muñoz-Tomás M, Manzano-Fernández $P$, Solsona-Hernández S, Mashat M, Gil-Gómez J. A novel virtual motor rehabilitation system for Guillain-Barré syndrome. Two single case studies. Methods Inf Med. 2015:54(2):127-34.

51. Kampshoff C, Jansen F, van Mechelen W, May A, Brug J, Chinapaw M, Buffart L. Determinants of exercise adherence and maintenance among cancer survivors: a systematic review. Int J Behav Nutr Phys Act. 2014;11:80.

52. C. Kampshoff, v. M. W. G. Schep, M. Nijziel, L. Witlox, L. Bosman, C. M.J, J. Brug and L. Buffart, "Participation in and adherence to physical exercise after completion of primary cancer treatment," Int J Behav Nutr Phys Act, vol. 13, no. 100,2016

53. Klompstra L, Jaarsma T, Strömberg A. Exergaming in older adults: a scoping review and implementation potential for patients with heart failure. Eur J Cardiovasc Nurs. 2014;13(5):388-98.

54. Robinson J, Dixon J, Macsween A, van Schaik P, Martin D. The effects of exergaming on balance, gait, technology acceptance and flow experience in people with multiple sclerosis: a randomized controlled trial. BMC Sports Sci Med Rehabil. 2015;7:8.

55. Mazzoleni S, Montagnani G, Vagheggini G, Buono L, Moretti F, Dario P, Ambrosino N. Interactive videogame as rehabilitation tool of patients with chronic respiratory diseases: preliminary results of a feasibility study. Respir Med. 2014;108(10):1516-24

56. Yong J, Soon Y, Xu D, Thia E, Pei Fen C, Kuah C, Kong K. A feasibility study using interactive commercial off-the-shelf computer gaming in upper limb rehabilitation in patients after stroke. J Rehabil Med. 2010;42(5):437-41.

57. Gutiérrez R, Galán Del Río F, Cano de la Cuerda R, Alguacil Diego I, González $\mathrm{R}$, Page J. A telerehabilitation program by virtual reality-video games improves balance and postural control in multiple sclerosis patients. NeuroRehabilitation. 2013;33(4):545-54.

58. Spruit M, Burtin C, De Boever P, Langer D, Vogiatzis I, Wouters E, Franssen F. COPD and exercise: does it make a difference? Breathe. 2016:12(2):e38-49.

59. Makhabah D, Suradi S, Doewes M. The role of interactive game-based system in pulmonary rehabilitation of patients with COPD. Eur Respir J. 2015:46:59.

60. Liao $Y$, Yang $Y$, Wu Y, Wang R. Virtual reality-based Wii fit training in improving muscle strength, sensory integration ability, and walking abilities in patients with Parkinson's disease: a randomized control trial. Int J Gerontology. 2015;9(4):190-5.

61. Saposnik G, Teasell R, Mamdani M, Hall J, Mcllroy W, Cheung D, Thorpe K, Cohen L, Bayley M. Effectiveness of virtual reality using Wii gaming Technology in Stroke Rehabilitation. Stroke. 2010;41(7):1477-84.

62. Ribas C, Alves da Silva L, Corrêa M, Teive H, Valderramas S. Effectiveness of exergaming in improving functional balance, fatigue and quality of life in Parkinson's disease: a pilot randomized controlled trial. Parkinsonism Relat Disord. 2017;38:13-8.

63. Brichetto G, Spallarossa P, Lopes de Carvalho M, Battaglia M. The effect of Nintendo ${ }^{\oplus}$ Wii $^{\oplus}$ on balance in people with multiple sclerosis: a pilot randomized control study. Mult Scler J. 2013;19(9):1219-21.

64. Meinhardt M, Hamid A, Schäfer J, Cruz D, Carvalho L, Gonçalves da Silva A. Effectiveness, safety and applicability of Wii therapy in patients with chronic obstructive pulmonary diseases. Journal of US-China Medical Science. 2013; 10(3-4):76-84.

65. Goldstein $\mathrm{R}$, Hill K, Brooks D, Dolmage T. Pulmonary rehabilitation: a review of the recent literature. Chest. 2012;142(3):738-49.

66. Maillot P, Perrot A, Hartley A. Effects of interactive physical-activity videogame training on physical and cognitive function in older adults. Psychol Aging. 2012;27(3):589-600.

67. Anderson-Hanley C, Arciero P, Brickman A, Nimon J, Okuma N, Westen S, Merz M, Pence B, Woods J, Kramer A, Zimmerman E. Exergaming and older adult cognition: a cluster randomized clinical trial. Am J Prev Med. 2012; 42(2):109-19. 
68. Rosenberg D, Depp C, Vahia I, Reichstadt J, Palmer B, Kerr J, Norman G, Jeste D. Exergames for subsyndromal depression in older adults: a pilot study of a novel intervention. Am J Geriatr Psychiatry. 2010;18(3):221-6.

69. Herz N, Mehta S, Sethi K, Jackson P, Hall P, Morgan J. Nintendo Wii rehabilitation ("Wii-hab") provides benefits in Parkinson's disease. Parkonsonism Relat Disord. 2013;19(11):1039-42.

70. Fung V, Ho A, Shaffer J, Chung E, Gomez M. Use of Nintendo Wii fit ${ }^{\text {tM }}$ in the rehabilitation of outpatients following total knee replacement: a preliminary randomised controlled trial. Physiotherapy. 2012;98(3):183-8.

71. Hsu J, Thibodeau R, Wong SZD, Cecile S, Walton D. A "Wii" bit of fun: the effects of adding Nintendo Wii( $\left(^{\circ}\right.$ bowling to a standard exercise regimen for residents of long-term care with upper extremity dysfunction. Physiother Theory Pract. 2011;27(3):185-93.

72. Collado-Mateo D, Merellano-Navarro E, Olivares P, García-Rubio J, Gusi N. Effect of exergames on musculoskeletal pain: a systematic review and metaanalysis. Scand J Med Sci Sports. 2017;28(3):1-12.

73. Gardner HR, Fraser C, MacLennan G, Treweek S. A protocol for a systematic review of non-randomised evaluations of strategies to improve participant recruitment to randomised controlled trials. Systematic Reviews. 2016;5:131.

74. Shrier S, Boivin JF, Steele RJ, Platt RW, Furlan A, Kakuma R, Brophy J, Rossignol M. Should meta-analyses of interventions include observational studies in addition to randomized controlled trials? A critical examination of underlying principles. Am J Epidemology. 2007;166(10):1203-9.

75. Arditi C, Burnand B, Peytremann-Bridevaux I. Adding non-randomised studies to a Cochrane review brings complementary information for healthcare stakeholders: an augmented systematic review and metaanalysis. BMC Health Serv Res. 2016;16:598.

Ready to submit your research? Choose BMC and benefit from:

- fast, convenient online submission

- thorough peer review by experienced researchers in your field

- rapid publication on acceptance

- support for research data, including large and complex data types

- gold Open Access which fosters wider collaboration and increased citations

- maximum visibility for your research: over $100 \mathrm{M}$ website views per year

At $\mathrm{BMC}$, research is always in progress.

Learn more biomedcentral.com/submissions 